SCIENTIFIC LETTER

\title{
Reliable detection of early myocardial dysfunction by tissue Doppler echocardiography in Becker muscular dystrophy
}

\author{
C Meune, O Pascal, H M Bécane, F Héloire, D Christoforou, P Laforet, B Eymard, P Gueret, \\ F Leturcq, D Recan, J Y Devaux, S Weber, D Duboc
}

Heart 2004;90:947-948. doi: 10.1136/hrt.2003.021113

B ecker muscular dystrophy (BMD) is an $\mathrm{X}$ linked mutation of the dystrophin gene characterised by skeletal muscle dystrophy and progressive heart failure which frequently leads to a fatal outcome. ${ }^{1}$ Cardiac involvement is not accurately predicted by gene mutation and may occur in patients without muscle weakness. Consequently, systematic cardiac examinations are required. Tissue Doppler echocardiography (TDE) allows accurate quantification of regional myocardial function and may be a reliable method for early detection of myocardial dysfunction in BMD. ${ }^{2}$

\section{METHODS}

Twenty four consecutive men with genetically documented BMD were prospectively investigated after informed consent and compared to 17 age matched controls. All patients underwent clinical examination, ECG, radionuclide ventriculography, and conventional echocardiography with TDE imaging.

Radionuclide ventriculography (gamma camera General Electric, Starport 400AT, interfaced with ADAC computer, DPS 3000) was performed after in vitro red cell labelling with $15 \mathrm{mCi}$ of technetium-99m.

Ultrasound examinations (HDI5000, ATL ultrasound, Bothell, Washington DC) were recorded and analysed offline (HDILab Software) by two cardiologists unaware of any information. Radial left ventricular (LV) thickening was interrogated using colour $\mathrm{M}$ mode TDE acquisitions in the posterior wall. Myocardial wall motion velocities were extracted in endocardium (EndoV) and epicardium (EpiV) in order to calculate peak systolic strain rate $\left(\mathrm{SR}_{\mathrm{SYS}}\right)$ and early diastolic strain rate $\left(S_{\text {DIA }}\right)$ with $S R=($ EndoV - EpiV $) /$ (distance between endocardium and epicardium). Interobserver variability was $0.04(0.4) / \mathrm{s}$ for $\mathrm{SR}_{\mathrm{SYS}}(r=0.91$, $\mathrm{p}<0.001)$ and $0.3(0.8) / \mathrm{s}$ for $\mathrm{SR}_{\mathrm{DIA}}(r=0.91, \mathrm{p}<0.001)$.

Data, expressed as median (interquartile range), were compared by the Kruskal-Wallis test followed by multiple comparisons using Mann-Whitney U test (multiple comparisons requiring $\mathrm{p}<0.0167$ for significance).

\section{RESULTS}

One patient with poor echographic imaging was excluded; results are therefore presented for 23 patients. The population was divided into three subgroups according to the radionuclide left ventricular ejection fraction (LVEF): group 1, 10 BMD patients (37.0 (10.8) years) with reduced LVEF $<55 \%$ (LVEF $43(8 \%)$ ); group 2, 13 BMD patients (25.6 (9.9) years) with normal LVEF 61(6\%); and group 3, 17 healthy controls (31.4 (6.6) years).

Heart rate and blood pressure did not differ between the three groups. Three patients in group 1 had dyspnoea (New York Heart Association functional class II and III). All had echocardiographic wall motion abnormalities, and the majority were treated with angiotensin converting enzyme (ACE) inhibitors or $\beta$ adrenergic blockers. In contrast, group 2 patients were free of symptoms and had normal cardiac examinations; two patients were treated with ACE inhibitors for hypertension.

As expected, compared to group 3, group 1 patients had lower $\mathrm{SR}_{\mathrm{SYS}}(1.7(0.8) / \mathrm{s} v 4.1(2.0) / \mathrm{s}$ respectively, $\mathrm{p}<0.001)$ and $\mathrm{SR}_{\text {DIA }}(2.8(1.5) / \mathrm{s} v 5.7(1.4) / \mathrm{s}, \mathrm{p}<0.001)$ (fig 1$)$.

All patients in group 2 had normal cardiac examinations, echocardiographic indices, and radionuclide LVEF. However, when compared to the control group they had lower $\mathrm{SR}_{\mathrm{SYS}}$ and $\mathrm{SR}_{\text {DIA }}(2.1(0.9) / \mathrm{s} v 4.1(2.0) / \mathrm{s}, \mathrm{p}=0.001$, and $3.5(1.6) / \mathrm{s}$ $v 5.7(1.4) / \mathrm{s}, \mathrm{p}=0.002$, respectively) (fig 1 ).

No significant difference was observed between group 1 and group $2\left(\mathrm{SR}_{\mathrm{SYS}}, \mathrm{p}=0.42\right.$ and $\left.\mathrm{SR}_{\mathrm{DIA}}, \mathrm{p}=0.321\right)$ (fig $\mathrm{l}$ ). Regarding individual values of $\mathrm{SR}$, seven patients in group 2 (normal LVEF) had $\mathrm{SR}_{\mathrm{SYS}}<2.0 / \mathrm{s}$ and six patients had $\mathrm{SR}_{\mathrm{DIA}}$ $<2.9 / \mathrm{s}$ (lowest values in controls), and 10 of these 13 patients had reduced $\mathrm{SR}_{\mathrm{SYS}}$ and/or $\mathrm{SR}_{\mathrm{DIA}}$.

\section{DISCUSSION}

Our study indicates that abnormal myocardial function may be present in patients with BMD, despite normal echocardiographic and radionuclide LVEF, pointing to the need for close follow up. TDE was also found to be more accurate and more sensitive than ultrasound and isotopic methods and should be considered in this setting.

Assessment of LV function in patients with suspected cardiomyopathy is commonly performed by echocardiography or radionuclide ventriculography. Routine measurements are, however, load dependent and do not systematically reflect the contractile state of the myocardium. In contrast, myocardial SR determined by TDE is known as an index of contractility independent of myocardial translational motion and loading variations, and is more sensitive than conventional indices. ${ }^{2} 3$ Using TDE in our study, we first confirmed the presence of LV systolic dysfunction in patients with a known decrease in LVEF and also demonstrated LV systolic dysfunction in BMD patients with normal LVEF. As $S_{\text {SYS }}$ and $\mathrm{SR}_{\mathrm{DIA}}$ are respective markers of myocardial systolic and diastolic function, these patients have both types of dysfunction. ${ }^{3}$ This is concordant with histological findings in BMD patients which have shown diffuse atrophy and fibrosis of both ventricles. ${ }^{1}$ Moreover, this complication seems to occur early in BMD (mean age in group 2 was 25.6 years), and is independent of the type of dystrophin gene

Abbreviations: $A C E$, angiotensin converting enzyme; $B M D$, Becker muscular dystrophy; LV, left ventricular; LVEF, left ventricular ejection fraction; $\mathrm{SR}_{\mathrm{DIA}}$, diastolic strain rate; $\mathrm{SR}_{\mathrm{SY}}$, systolic strain rate; $T D E$, tissue Doppler echocardiography 

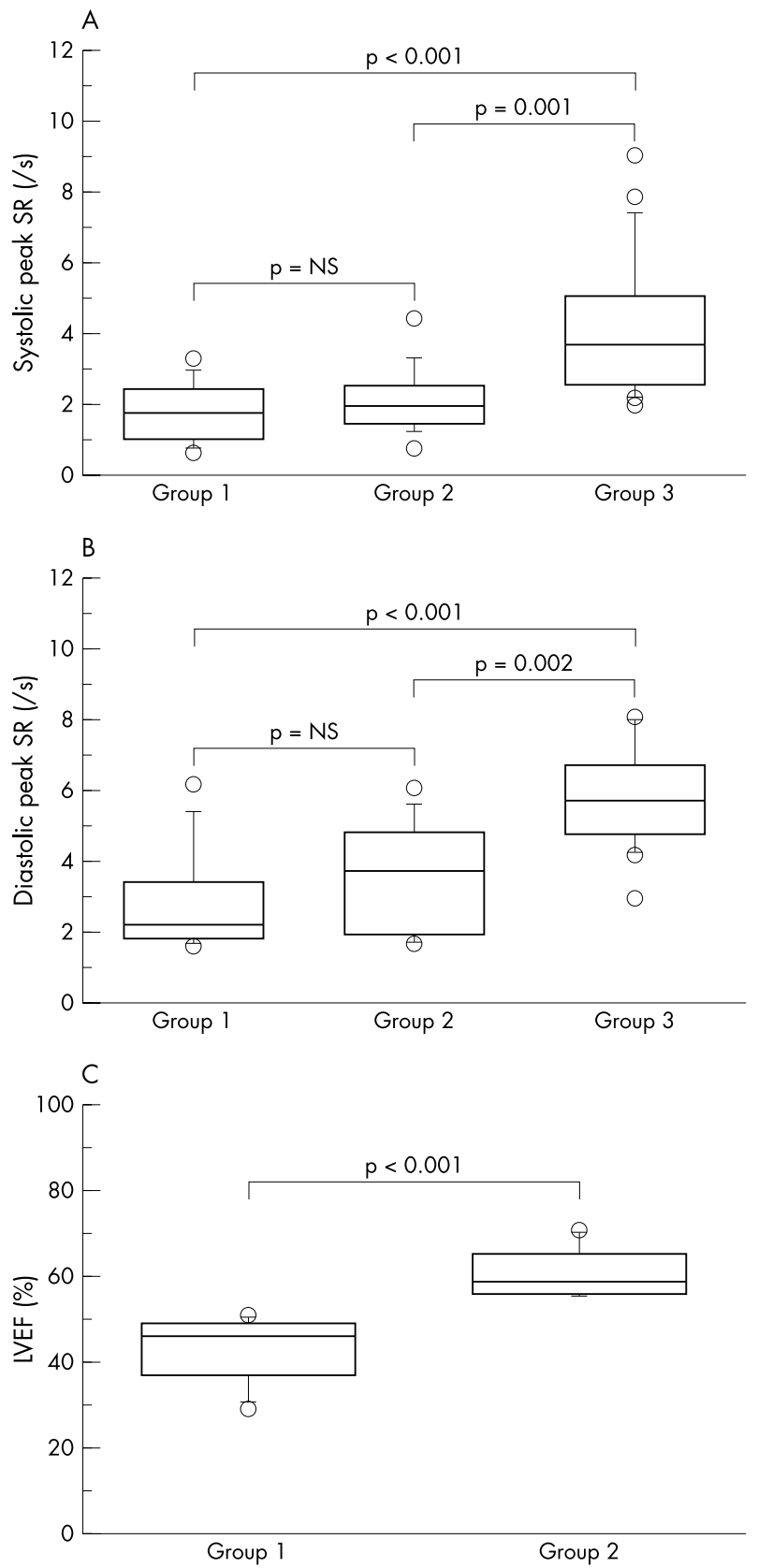

Figure 1 (A) SR $R_{S Y S},(B) S R_{D I A}$, and (C) LVEF, in patients with left ventricular (LV) dysfunction (group 1), without LV dysfunction (group 2), and in healthy subjects (group 3). mutation/deletion (data not shown). ${ }^{4}$ Combined with the possibility of a complete dissociation between muscle and myocardial involvement, this further highlights the need for appropriate and accurate detection of myocardial dysfunction.

This may have important clinical implications. The detection of systolic dysfunction is known as a crucial issue for the prognostic evaluation of patients with cardiomyopathy associated with muscular diseases. ${ }^{5}$ Since intense exercise may exacerbate myocardial damage via increases in LV pressure and volume overload, our patients were recommended to abstain from engaging in strenuous physical activities. Moreover, the early use of afterload reducing drugs (ACE inhibitors) may provide effective prevention and warrants study.

BMD patients, with either normal or decreased LVEF, may have myocardial systolic and diastolic dysfunction detected by TDE. This method may offer new diagnostic and therapeutic opportunities in the screening and management of these patients, and should be considered in these settings.

Authors' affiliations

C Meune, F Héloire, D Christoforou, S Weber, D Duboc, Department of Cardiology, Cochin Hospital, René Descartes University, Paris, France O Pascal, P Gueret, Department of Cardiology, Henri-Mondor Hospital, Créteil, France

H M Bécane, P Laforet, B Eymard, Myology Institute, Pitié-la Salpétrière Hospital, Paris

F Leturcq, D Recan, Genetic Institute, Cochin Hospital, René Descartes University, Paris

J Y Devaux, Nuclear Medicine, Cochin Hospital

Correspondence to: Dr Christophe Meune, Department of Cardiology, Cochin Hospital, 27 rue du Faubourg St-Jacques, 75014 Paris, France; christophe.meune@cch.ap-hop-paris.fr

Accepted 27 October 2003

\section{REFERENCES}

1 Melacini P, Fanin M, Danieli GA, et al. Cardiac involvement in Becker muscular dystrophy. J Am Coll Cardiol 1993;22:1927-34.

2 Uematsu M, Nakatani S, Yamagishi M, et al. Usefulness of myocardial velocity gradient derived from two-dimensional tissue Doppler imaging as an indicator of regional myocardial contraction independent of translational motion assessed in atrial septal defect. Am J Cardiol 1997:79:237-41.

3 Shimizu $Y$, Uematsu $M$, Shimizu $H$, et al. Peak negative myocardial velocity gradient in early diastole as a noninvasive indicator of left ventricular diastolic function: comparison with transmitral flow indices. J Am Coll Cardiol 1998;32:1418-25

4 Steare SA, Dubowitz V, Benatar A. Subclinical cardiomyopathy in Becker muscular dystrophy. Br Heart J 1992;68:304-8.

5 Corrado G, Lissoni A, Beretta S, et al. Prognostic value electrocardiograms, ventricular late potentials, ventricular arrhythmias, and left ventricular systolic dysfunction in patients with Duchenne muscular dystrophy. Am J Cardiol 2002;89:838-41 